\title{
A longitudinal study investigating changes in the expression of efflux transporters in the lactating mammary epithelial cells and their impact on the transfer of drugs from mother to breastfed infant via breastmilk
}

\author{
Hilai Ahmadzai, Lisa Bg Tee, Richard Parsons, Andrew P Crowe
}

School of Pharmacy and Biomedical Science, Curtin University, Australia

Background: Although breastfeeding is advocated as the best nutritional start for an infant, there is always concern regarding the transfer of medications from mother to their breastfed baby via milk. While most drugs are compatible with breastfeeding, cases of toxic drug exposure have been reported. This is thought to be due to active transport mechanisms whereby efflux transporter proteins expressed in the epithelial cells of the mammary gland actively secrete drugs into milk. An example of such efflux transporters is the breast cancer resistance protein (BCRP) which is strongly induced during lactation and this could result in contamination of milk with the substrates of this transporter which may place the suckling infant at risk of toxicity.

Aim: The objective of this study was to investigate the changes in the expression of four ATPase Binding Cassette (ABC) transporters including BCRP, MDR1, MRP1 and MRP2 in the lactating human mammary epithelial cells at various time points during lactation and to explore whether cells derived from breastmilk can be used to develop an individualised, non-invasive model to predict drug transfer from mother to baby via breastmilk.

Methods: Milk samples were collected from nursing mothers at various time points starting at one month post-partum (intended at 1, 3, 5, 9 and 12 months) until a maximum of 12 months post-partum or cessation of breastfeeding. Gene expression of the transporters was tested both at the mRNA (qRT-PCR) and the protein levels (immunostaining). Epithelial cells isolated from freshly expressed breastmilk were isolated and successfully grown in a specialised medium.

Results: Breastmilk cell gene expression varied widely amongst participants. Our results indicate that there is a strong trend showing a monthly increase of +2.24 in MRP2 $(\mathrm{p}=0.0002)$, and a weaker (but significant) increase in MDR1 $(+0.17$ per month; $\mathrm{p}=0.0102)$. There was no evidence of a change in BCRP or MRP1 over time. The expression of these transporters also varied significantly in cultured cells compared to fresh cells.

Conclusion: Our results show that the expression of some ABC transporters is stage dependent in humans and may exhibit some intersubjective variability. 